Publ. RIMS, Kyoto Unit.

18 (1982), $1-29$

\title{
Invertibility for Microdifferential Operators of Infinite Order
}

By

\author{
Takashi AOKI*
}

\begin{abstract}
Alustract
For each holomorphic microlocal operator its symbol is defined as a holomorphic function which satisfies a growth condition. A differential or micro(=pseudo-) differential operator of infinite order is naturally regarded as a holomorphic microlocal operator and its symbol coincides with the usual total symbol. Some sufficient conditions of invertibility for holomorphic microlocal operators in terms of symbols are given.
\end{abstract}

\section{Introduction}

The sheaf of rings $\mathcal{E}^{\boldsymbol{R}}$ is defined by Sato, Kawai, and Kashiwara (cf. [7], [10]). It plays important roles in the studies of many problems (for example, see [2], [3], [4], [5]). The sheaf $\mathcal{E}^{\mathbb{R}}$ includes differential operators of infinite order, micro(=pseudo-) differential operators of infinite order and of fractional order. We tentatively call a section of $\mathcal{E}^{\mathbb{R}}$ a holomorphic microlocal operator (cf. [7]), even though there has not yet been a common agreement on naming. In this paper, we study invertibility for holomorphic microlocal operators.

Ellipticity and microlocal ellipticity for differential operators of infinite order with constant coefficients have been investigated by Kawai [8]. We consider microlocal ellipticity for an operator as invertibility in the ring of holomorphic microlocal operators. In this sense we extend his results to variable coefficient case.

Set $X=\mathbb{C}^{n} \ni\left(x_{1}, \cdots, x_{n}\right)$ and $T^{*} X^{*} \cong \mathbb{C}^{n} \times \mathbb{C}^{n} \ni\left(x_{1}, \cdots, x_{n} ; \zeta_{1}, \cdots, \zeta_{n}\right)$ $=(x, \zeta)$. We use the notation:

$$
D_{x}^{\alpha}=D_{x_{1}}^{\alpha_{1}} \cdots \cdots D_{x_{n}}^{\alpha_{n}}=\left(\frac{\partial}{\partial x_{1}}\right)^{\alpha_{1}} \cdots\left(\frac{\partial}{\partial x_{n}}\right)^{\alpha_{n}}
$$

Communicated by S. Matsuura, May 20, 1980.

* Department of Mathematics, Faculty of Science, The University of Tokyo, Bunkyo-ku, Tokyo 113, Japan. 


$$
\begin{aligned}
& \zeta=\zeta_{1}{ }^{\alpha_{1}} \cdots \cdots \zeta_{n}{ }^{\alpha_{n}}, \\
& |\alpha|=\alpha_{1}+\cdots+\alpha_{n},
\end{aligned}
$$

and

$$
\alpha !=\alpha_{1} ! \cdots \cdot \alpha_{n} !,
$$

where $\alpha=\left(\alpha_{1}, \cdots, \alpha_{n}\right) \in \mathbb{Z}_{+}^{n} \quad\left(\boldsymbol{Z}_{+}=\{0,1,2, \cdots\}\right)$. Let us denote by $\mathcal{E}_{X}^{\mathbb{R}}$ (resp. $\mathcal{E}_{X}^{\infty}$ ) the sheaf on $T^{*} X$ of holomorphic microlocal operators (resp. microdifferential operators of infinite order) and by $\mathscr{D}_{X}^{\infty}$ the sheaf on $X$ of differential operators of infinite order. There are canonical injections $\pi^{-1} \mathscr{D}_{X}^{\infty} \rightarrow \mathcal{E}_{X}^{\infty}$ and $\mathcal{E}_{X}^{\infty} \rightarrow \mathcal{E}_{X}^{R}$, where $\pi: T^{*} X \rightarrow X$ is the projection. Let $\dot{x}^{*}$ be a point in $T^{*} X$. For a holomorphic microlocal operator $F \in \mathcal{E}_{X, x^{*}}^{\mathbb{R}}$, its symbol $F(x, \zeta)$ is a holomorphic function defined in some conic neighborhood $\Gamma$ of $\dot{x}^{*}$ which satisfies the following estimate in $\Gamma$ : for each $\delta>0$ there is a constant $C$ such that $|F(x, \zeta)| \leqq C \exp (\delta|\zeta|)$ (cf. [6]). Then the operator $F$ is expressed as $F\left(x, D_{x}\right)$. This notation is justified by the composition rule in terms of symbols.

In our setting, Kawai's theorem on microlocal ellipticity for differential operators of infinite order with constant coefficients ([8] Theorem 4.1.8) implies the following: Let $P\left(D_{x}\right)=\sum_{|\alpha| \geq 0} a_{\alpha} D_{x}{ }^{\alpha}$ be a differential operator of infinite order with constant coefficients. Let $P(\zeta)=\sum_{|\alpha| \geq 0} a_{\alpha} \zeta^{\alpha}$ be its total symbol. Set $V=\left\{\zeta \in C^{n} ; P(\zeta)=0\right\}$. Let $\zeta_{0} \in \mathbb{C}^{n}$ and assume that there is a conic neighborhood $\Gamma$ of $\zeta_{0}$ such that

$$
\Gamma \cap V \cap\{\zeta ;|\zeta|>R\}=\varnothing \text { for some } R>0 .
$$

Then $P\left(D_{x}\right)$ is invertible as an operator in $\varepsilon_{X, x^{*}}^{\boldsymbol{R}}$ for each $\dot{x}^{*}=\left(x, \zeta_{0}\right)$.

One of the most essential part in his proof is to obtain the estimate for $|1 / P(\zeta)|$ from (0.5). That is, for any $\varepsilon>0$ there is $C^{\prime}>0$ such that $|P(\zeta)| \geqq C^{\prime} \exp (-\varepsilon|\zeta|)$. In his proof Kawai essentially uses the fact that $P(\zeta)$ is an entire function of order 1 of minimal type. The symbol of a holomorphic microlocal operator is not an entire function in general, however. Hence we cannot expect the estimate done by Kawai. Therefore we begin our discussion with assuming some estimate for the symbol from below. In our case such an estimate does not immediately imply the invertibility of the operator except for the case where the operator in question is with constant coefficients. Actually we still find some technical 
difficulties in proving our final result in the general case, and we content ourselves with studying operators whose symbols satisfy an extra growth condition specified below. Roughly speaking we deal with operators which naturally appear in connection with ultradistributions of class (s) for $2 \leqq s \leqq \infty$ (cf. [9]).

We prove the following theorem: Let $P$ be a holomorphic microlocal operator in $\mathcal{E}_{X, \hat{x}^{*}}^{\mathbb{R}}$ with its symbol $P(x, \zeta)$ defined in some conic neighborhood $\Gamma$ of $\dot{x}^{*}$. Let $\rho$ be a number such that $0 \leqq \rho \leqq 1 / 2$. Assume that there exist positive constants $h, C_{0}, C_{1}$ such that

$$
C_{0} \exp \left(-h|\zeta|^{\rho}\right) \leqq|P(x, \zeta)| \leqq C_{1} \exp \left(h|\zeta|^{\rho}\right) \quad \text { for } \quad(x, \zeta) \in \Gamma
$$

Then $P$ is invertible in the ring $\mathcal{E}_{X, x^{*}}^{\mathbb{R}}$. The inverse $U$ of $P$ is given by $U=Q R$ where $Q$ is a holomorphic microlocal operator with symbol $(P(x, \zeta))^{-1}$ and $R$ is an operator of order 0 . The principal part of $R$ is

$$
\exp \left(\sum_{j=1}^{n} \partial_{\zeta,} p(x, \zeta) \cdot \partial_{x_{j}} p(x, \zeta)\right)
$$

where $p(x, \zeta)=\log P(x, \zeta)$. This is a natural extension of the theorem on microlocal ellipticity for micro(=pseudo-) differential operators of finite order ([10] Chapter II, Theorem 2.1.1).

Let $M=\mathbb{R}^{n}$ with its complexification $X=\mathbb{C}^{n}$ and $T_{M}^{*} X$ the conormal bundle of $M$ in $T^{*} X$. Let us denote by $\mathscr{C}_{M}$ the sheaf on $T_{M}^{*} X$ of

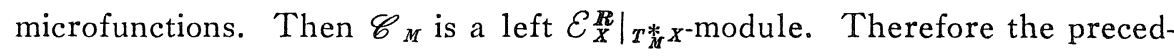
ing theorem yields the following theorem: Let $\dot{x}^{*} \in T_{M}^{*} X$ and $P$ be an operator in $\left.\mathcal{E}_{X}^{R}\right|_{\dot{x}^{*}}$ satisfying the condition of the preceding theorem. Then the mapping

$$
P: \mathscr{C}_{M, \dot{x}^{*}} \rightarrow \mathscr{C}_{M, \dot{x}^{*}}
$$

is bijective.

The plan of this paper is as follows:

In $\S 1$, we give the notation.

In $\S 2$, we define the symbols of holomorphic microlocal operators and establish some calculation rules for holomorphic microlocal operators in terms of symbols. In addition, we define the growth order of holomorphic microlocal operators.

In $\S 3$, we study invertibility for holomorphic microlocal operators. 


\section{$\S$ 1. Preliminaries}

1. 1. Let $X$ be an $n$-dimensional complex manifold and $T^{*} X$ be the cotangent vector bundle of $X$, Let us denote by $\mathcal{O}_{X}$ the sheaf on $X$ of holomorphic functions and by $\Omega_{X}^{n}$ the sheaf of holomorphic $n$-forms on $X$. We identify $X$ with the diagonal of $X \times X$ and $T^{*} X$ with the conormal bundle $T_{X}^{*}(X \times X)$ of $X$ in $T^{*}(X \times X)$. We denote by $\left.\mathscr{C}_{X}^{R}\right|_{X \times X}$ the sheaf of holomorphic microfunctions on $T_{X}^{*}(X \times X)$ (see Sato-KawaiKashiwara [10]).

The sheaf $\mathcal{E}_{X}^{\mathbb{R}}$ of rings of holomorphic microlocal operators on $T^{*} X$ is given by

$$
\mathcal{E}_{X}^{\boldsymbol{R}}=\mathscr{C}_{X \mid X \times X}^{\boldsymbol{R}} \bigotimes_{p_{2}^{-1} \mathcal{O}_{X}} p_{2}^{-1} \Omega_{X}^{n}
$$

where $p_{2}: T_{X}^{*}(X \times X) \rightarrow X \times X \rightarrow X$ denotes the second projection. We will call a section of $\mathcal{E}_{X}^{\mathbb{R}}$ a holomorphic microlocal operator.

Let us recall that the sheaf $\mathcal{E}_{X}^{\infty}$ of microdifferential operators on $T^{*} X$ is given by

$$
\begin{aligned}
& \left.\mathcal{E}_{X}^{\infty}\right|_{\left(T^{*} X-X\right)}=\gamma^{-1} \gamma_{*} \mathcal{E}_{X}^{\boldsymbol{R}}, \\
& \left.\mathcal{E}_{X}^{\infty}\right|_{X}=\left.\mathcal{E}_{X}^{R}\right|_{X},
\end{aligned}
$$

where $r$ is the projection on the complex projective cotangent bundle:

$$
r: T^{*} X-T_{X}^{*} X \rightarrow P^{*} X=\left(T^{*} X-T_{X}^{*} X\right) / \mathbb{C}^{*} .
$$

We have canonical injections

$$
\pi^{-1} \mathscr{D}_{X}^{\infty} \hookrightarrow \mathcal{E}_{X}^{\infty}, \quad \mathcal{E}_{X}^{\infty} \hookrightarrow \mathcal{E}_{X}^{R}
$$

and

$$
\left.\mathcal{E}_{X}^{\mathbb{R}}\right|_{X}=\left.\mathcal{E}_{X}^{\infty}\right|_{X}=\mathscr{D}_{X}^{\infty}\left(X \simeq T_{X}^{*} X\right),
$$

where $\mathscr{D}_{X}^{\infty}$ is the sheaf on $X$ of differential operators (of infinite order) and $\pi$ is the projection

$$
\pi: T^{*} X \rightarrow X
$$

The sheaf $\mathcal{E}_{X}^{\boldsymbol{R}}$ (resp. $\mathcal{E}_{X}^{\infty}, \mathscr{D}_{X}^{\infty}$ ) is often abbreviated to $\mathcal{E}^{\boldsymbol{R}}$ (resp. $\left.\mathcal{E}^{\infty}, \mathscr{D}^{\infty}\right)$.

1.2. A subset $\Gamma$ in $T^{*} X$ is called conic if each fiber $\Gamma \cap\left(T^{*} X\right)_{x}$ 
is a cone in $\left(T^{*} X\right)_{x} \simeq \mathbb{C}^{n} \simeq \mathbb{R}^{2 n}(x \in X)$. A conic subset $\Gamma^{\prime}$ in $T^{*} X$ is said to be compact if $\gamma_{\boldsymbol{R}}(\Gamma)$ is compact where

$$
\gamma_{\mathbb{R}}: T^{*} X-T_{X}^{*} X \rightarrow S^{*} X=\left(T^{*} X-T_{\mathscr{X}}^{*} X\right) / \mathbb{R}^{+}
$$

is the projection. We often neglect a bounded subset of a conic subset in $T^{*} X$.

Remark. We denote by $\mathcal{E}_{X}^{\boldsymbol{R}}$ and $\mathcal{E}_{X}^{\infty}$ the sheaves denoted by $\mathscr{Q}_{X}^{\mathbb{R}}$ in [7] ( $\mathcal{L}^{\boldsymbol{C}}$ in [6]) and $\mathscr{L}_{X}$ (on $P^{*} X$ ) in [7], [10] respectively as this notation is now more commonly used. We also call the sections of $\mathcal{E}_{X}^{\infty}$ microdifferential operators instead of pseudo-differential operators.

\section{$\S 2.5 y m b o l s$ of Holomorphic Microlocal Operators}

2. 1 . Let $X$ be a complex manifold of dimension $n$ and take a point $\dot{x}^{*}$ in the cotangent bundle $T^{*} X$. From now on, we fix a local coordinate system $x=\left(x_{1}, \cdots, x_{n}\right)$ of $X$. Let $(x, \zeta)=\left(x_{1}, \cdots, x_{n} ; \zeta_{1}, \cdots, \zeta_{n}\right)$ be coordinates of $T^{*} X$, where $\zeta=\left(\zeta_{1}, \cdots, \zeta_{n}\right)$ is cotangent coordinates. Without loss of generality, we can assume that $\dot{x}^{*}=(0 ; \lambda, 0, \cdots, 0)$, where $\lambda$ is a complex number. If $\lambda=0$, we have $\mathcal{E}_{\dot{x}^{r}}^{\boldsymbol{R}}=\mathscr{D}_{\tilde{x}^{*}}^{\infty}$. Since the symbols of differential operators are well known, we assume that $\lambda \neq 0$. The ring $\mathcal{E}_{\dot{x}^{*}}^{\boldsymbol{R}}$ is defined as the inductive limit

$$
\mathcal{E}_{\tilde{x}^{*}}^{\boldsymbol{R}}=\underset{c, \varepsilon}{\lim } H_{Z_{c, \varepsilon}}^{n}\left(U_{C} ; \circlearrowleft^{(0, n)}\right),
$$

where we set

$$
U_{c}=\left\{\left(x, x^{\prime}\right) \in X \times X ;|x|<c,\left|x-x^{\prime}\right|<c\right\},
$$

$$
\begin{gathered}
Z_{c, \varepsilon}=\left\{\left(x, x^{\prime}\right) \in U_{c} ; \operatorname{Re}\left(\lambda\left(x_{1}-x_{1}{ }^{\prime}\right)\right) \geqq \varepsilon\left|\operatorname{Im}\left(\lambda\left(x_{1}-x_{1}{ }^{\prime}\right)\right)\right|,\right. \\
\left.\left|x_{1}-x_{1}{ }^{\prime}\right| \geqq \varepsilon\left|x_{j}-x_{j}{ }^{\prime}\right|, j=2, \cdots, u^{\prime}\right\}
\end{gathered}
$$

for $c>0, \varepsilon>0$. Here $\mathcal{O}^{(0, n)}=\mathcal{O}_{X \times X} \underset{p_{2}{ }^{-1} \mathcal{O}_{X}}{\bigotimes} p_{2}^{-1} \Omega_{X}^{n}$ is the sheaf of $n$-forms with respect to the second variable.

Let us fix $c, \varepsilon$ and write $U=U_{c}, Z=Z_{c, \varepsilon}$. There is a homomorphism

$$
H_{Z}^{n}\left(U ; \mathcal{O}^{(0, n)}\right) \rightarrow \mathcal{E}_{\dot{x}^{*}}^{\mathbb{R}} .
$$

The open set $U-Z$ is covered by holomorphically convex sets $V^{(\nu)}$ 
$(\nu=1,2, \cdots, n)$ defined by

$$
\begin{aligned}
& V^{(1)}=\left\{\left(x, x^{\prime}\right) \in U ; \operatorname{Re}\left(\lambda\left(x_{1}-x_{1}^{\prime}\right)\right)<\varepsilon\left|\operatorname{Im}\left(\lambda\left(x_{1}-x_{1}^{\prime}\right)\right)\right|\right\}, \\
& V^{(\nu)}=\left\{\left(x, x^{\prime}\right) \in U ;\left|x_{1}-x_{1}^{\prime}\right|<\varepsilon\left|x_{\nu}-x_{\nu}^{\prime}\right|\right\} \text { for } \nu=2, \cdots, n .
\end{aligned}
$$

Set

$$
V=\bigcap_{\nu=1}^{n} V^{(\nu)}, \quad \widehat{V}^{(\nu)}=\bigcap_{\mu \neq \nu} V^{(\mu)} .
$$

We have the exact sequence

$$
\bigoplus_{\nu=1}^{n} \Gamma\left(\widehat{V}^{(\nu)} ; \mathcal{O}^{(0, n)}\right) \rightarrow \Gamma\left(V ; \mathcal{O}^{(0, n)}\right) \rightarrow H_{Z}^{n}\left(U ; \mathcal{O}^{(0, n)}\right) \rightarrow 0 .
$$

Combining this with (2.1.3) yields a mapping

$$
\Gamma\left(V ; \mathcal{O}^{(0, n)}\right) \rightarrow H_{Z}^{n}\left(U ; \mathcal{O}^{(0, n)}\right) \rightarrow \mathcal{E}_{\dot{x}^{*}}^{\boldsymbol{R}}
$$

Let $K\left(x, x^{\prime}\right)$ be a holomorphic function defined on $V$. The operator in $\mathcal{E}_{\dot{x}^{*}}^{\boldsymbol{R}}$ corresponding to $K\left(x, x^{\prime}\right) d x^{\prime} \in \Gamma\left(V ; \mathcal{O}^{(0, n)}\right)$ is denoted by $F$.

Set $L(x, y)=K(x, x-y)$, then $L$ is holomorphic on $\left\{(x, y) \in \mathbb{C}^{n}\right.$ $\left.\times C^{n} ;|x|<c,|y|<c, \operatorname{Re}\left(\lambda y_{1}\right)<\varepsilon\left|\operatorname{Im}\left(\lambda y_{1}\right)\right|,\left|y_{1}\right|<\varepsilon\left|y_{j}\right|, j=2, \cdots, n\right\}$. Let $\alpha_{0}$, $\alpha_{1}$ be two points sufficiently near $y_{1}=0$ such that

$$
0<-\varepsilon_{1} \operatorname{Im}\left(\lambda \alpha_{0}\right)<\operatorname{Re}\left(\lambda \alpha_{0}\right)<-\varepsilon \operatorname{Im}\left(\lambda \alpha_{0}\right)
$$

and that

$$
0<\varepsilon_{1} \operatorname{Im}\left(\lambda \alpha_{1}\right)<\operatorname{Re}\left(\lambda \alpha_{1}\right)<\varepsilon \operatorname{Im}\left(\lambda \alpha_{1}\right),
$$

where $0<\varepsilon_{1}<\varepsilon$.

Set

$$
k(x, \zeta, p)=\frac{(n-1) !}{(-2 \pi \sqrt{-1})^{n}} \int_{r_{1} \times \cdots \times r_{n}} \frac{L(x, y)}{(p-\langle y, \zeta\rangle)^{n}} d y,
$$

where $\langle y, \zeta\rangle=y_{1} \zeta_{1}+\cdots+y_{n} \zeta_{n}$ and $\gamma_{j}$ are paths determined as follows:
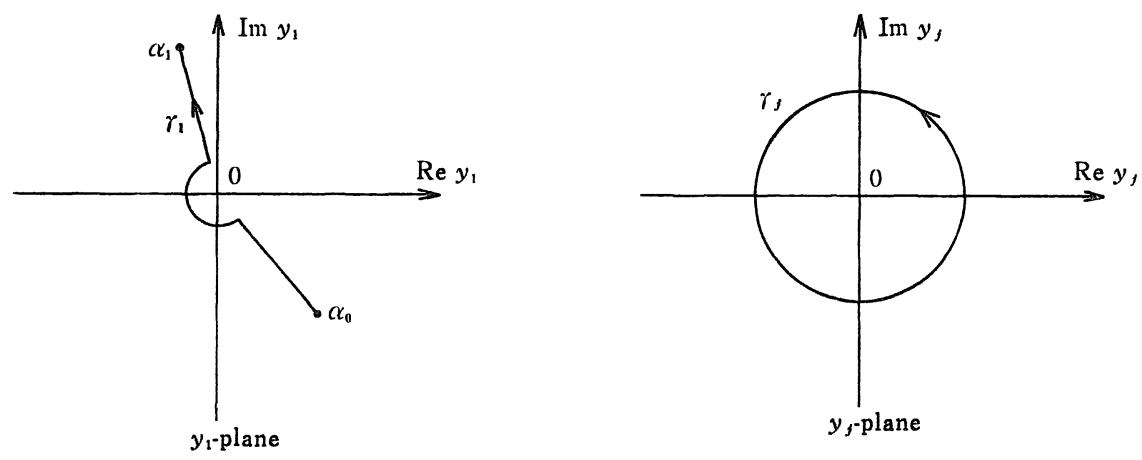
$\gamma_{1}$ is a path starting from $\alpha_{0}$, ending at $\alpha_{1}$ and around the origin clockwise, and $\gamma_{j}(j \geqq 2)$ is a cycle rounding $y_{j}=0$ counterclockwise with radius $>\varepsilon^{-1}\left|y_{1}\right|$. Then $k(x, \zeta, p)$ is holomorphic on (2.1.6) $D=\left\{(x, \zeta, p) \in \mathbb{C}^{n} \times \mathbb{C}^{n} \times \mathbb{C} ;|x|<c, \operatorname{Re}\left(\lambda^{-1} \zeta_{1}\right)>a\left|\operatorname{Im}\left(\lambda^{-1} \zeta_{1}\right)\right|\right.$,

$$
a\left|\zeta_{j}\right|<\left|\zeta_{1}\right| \quad(j=2, \cdots, n)
$$$$
\left.\operatorname{Re} p<\varepsilon_{1}|\operatorname{Im} p|, a|p|<|\zeta|\right\}
$$

for some positive constant $a$. Moreover, $k(x, \zeta, p)$ is homogeneous with respect to $(\zeta, p)$ of order $(-n)$ 。

Let us remark that $k\left(x, \zeta,\left\langle x-x^{\prime}, \zeta\right\rangle\right) \omega(\zeta) d x^{\prime}$ is nothing but the normalized Radon transformation of $F$ where

$$
\omega(\zeta)=\sum_{j=1}^{n}(-1)^{j-1} \zeta_{j} d \zeta_{1} \wedge \cdots \wedge \widehat{d \zeta_{j}} \wedge \cdots \wedge d \zeta_{n}
$$

(See Kataoka [6], [7]). If $K\left(x, x^{\prime}\right) d x^{\prime}$ belongs to the zero class, that is $F=0$ in $\mathcal{E}_{\tilde{x}^{*}}^{\mathbb{R}}$, then $k(x, \zeta, p)$ is holomorphic at $p=0$. The $k(x, \zeta, p)$ is independent of $\alpha_{j}(j=0,1)$ modulo holomorphic functions at $p=0$.

Theorem 2.1.1. Lct $s_{0}, s_{1}$ be two holomorphic functions of $\zeta$ of homogeneous of degree 1 defined on

$$
\left\{\zeta \in \mathbb{C}^{n} ; \operatorname{Re}\left(\lambda^{-1} \zeta_{1}\right)>a\left|\operatorname{Im}\left(\lambda^{-1} \zeta_{1}\right)\right|, a\left|\zeta_{j}\right|<\left|\zeta_{1}\right| \quad(j=2, \cdots, n)\right\}
$$

such that

$$
0<\operatorname{Re} s_{0}(\zeta)<-\varepsilon_{1} \operatorname{Im} s_{0}(\zeta), \quad a\left|s_{0}(\zeta)\right|<|\zeta|
$$

and that

$$
0<\operatorname{Re} s_{1}(\zeta)<\varepsilon_{1} \operatorname{Im} s_{1}(\zeta), \quad a\left|s_{1}(\zeta)\right|<|\zeta| .
$$

Set

$$
F(x, \zeta)=(2 \pi \sqrt{-1})^{n-1} \int_{\sigma(\zeta)} k(x, \zeta, p) \exp (-p) d p
$$

where the path $\sigma(\zeta)$ of integration is taken as figure. 


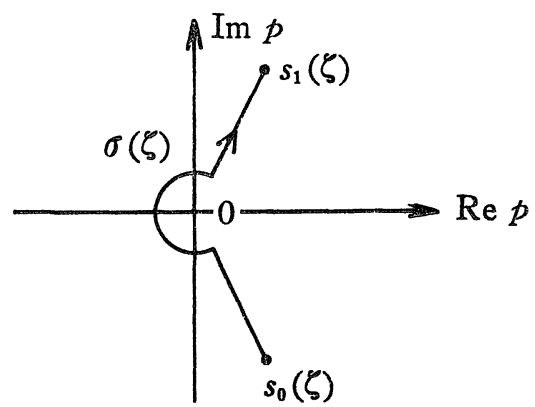

Then we have the following:

(a) $F(x, \zeta)$ is holomorphic on

$$
\Gamma=\left\{(x, \zeta) \in C^{n} \times C^{n} ;\right.
$$$$
\left.|x|<c, \operatorname{Re}\left(\lambda^{-1} \zeta_{1}\right)>a\left|\operatorname{Im}\left(\lambda^{-1} \zeta_{1}\right)\right|, a\left|\zeta_{j}\right|<\left|\zeta_{1}\right| \quad(j=2, \cdots, n)\right\} .
$$

(b) For every compact cone $\Gamma^{\prime} \mathbb{C} \Gamma$ and for every $\delta>0$,

$$
\sup _{\Gamma^{\prime}}|F(x, \zeta) \exp (-\delta|\zeta|)|<\infty .
$$

(c) Assume that $k(x, \zeta, p)$ is holomorphic at $p=0$, then for each compact cone $\Gamma^{\prime} \mathbb{E} \Gamma$, there exists a positive number $\delta_{0}$ such that

$$
\sup _{r^{\prime}}\left|F(x, \zeta) \exp \left(\delta_{0}|\zeta|\right)\right|<\infty
$$

Proof. By the definition of $F(x, \zeta)$, (a) is clear.

Let $\delta$ be a positive number. Take $\delta^{\prime}$ as $0<\delta^{\prime}<\delta$. We can take the path of integration as it is contained in $\left\{\operatorname{Re} p>-\delta^{\prime}|\zeta|\right\}$. Thus we have (b).

If $k(x, \zeta, p)$ is holomorphic on $\left\{|p|<\delta_{1}|\zeta|\right\}$ for some $\delta_{1}>0$, we can choose the path $\sigma$ of integration as it is contained in $\left\{\operatorname{Re} p>\delta_{1}^{\prime}|\zeta|\right\}$ for some $0<\delta_{1}^{\prime}<\delta_{1}$. If we take $\delta_{0}$ as $\delta_{0}<\delta_{1}^{\prime}$, we have (c).

A holomorphic function which satisfies the estimate (2.1.8) (resp. (2.1.9)) is called infra-exponential type (resp. rapidly decreeasing type).

We give the definition of symbols of holomorphic microlocal operators in accordance with Kataoka [6]. 
Definition 2. 1.2. The holomorphic function $F(x, \zeta)$ obtained in the preceding theorem is called the symbol defined in $\Gamma$ of the holomorphic microlocal operator $F \in \mathcal{E}_{\dot{x}^{*}}^{\mathbb{R}}$.

Then $F$ is represented as $F\left(x, D_{x}\right)$ where $D_{r}=\left(\frac{\partial}{\partial x_{1}}, \cdots, \frac{\partial}{\partial x_{n}}\right)$.

The symbol of the holomorphic microlocal operator is independent of $s_{j}(j=0,1)$ modulo rapidly decreasing functions. When $F=\sum_{|\alpha| \sum_{i}} a_{a}(x) D_{x}^{\alpha}$ is a differential operator, the symbol of $F$ in the sense of the definition coincides with its ordinary total symbol $F(x, \zeta)=\sum_{|\alpha| \geq 0} a_{a}(x) \zeta^{\alpha}$.

Theorem 2.1.3. Let $G(x, \zeta)$ be a holomorphic function of infra-exponential type defined on

$$
\begin{aligned}
\Gamma=\left\{(x, \zeta) \in \mathbb{C}^{n} \times \mathbb{C}^{n} ;|x|<c,|\zeta|>r,\right. \\
\left.\quad \operatorname{Re}\left(\lambda^{-1} \zeta_{1}\right)>a\left|\operatorname{Im}\left(\lambda^{-1} \zeta_{1}\right)\right|, a\left|\zeta_{j}\right|<\left|\zeta_{1}\right| \quad(j=2, \cdots, n)\right\}
\end{aligned}
$$

where $c>0$ is a small number, $a$ and $r$ are large positive numbers.

Set

$$
g(x, \zeta, p)=\frac{1}{(2 \pi \sqrt{-1})^{n}} \int_{R}^{+\infty} G(x, \tau \zeta) \exp (\tau p) \tau^{n-1} d \tau
$$

for $\zeta_{1}=\lambda$ where $R \gg r$ is a constant, and

$$
g(x, \zeta, p)=\left(\frac{\lambda}{\zeta_{1}}\right)^{n} g\left(x, \frac{\lambda}{\zeta_{1}} \zeta, \frac{\lambda}{\zeta_{1}} p\right)
$$

for $\zeta_{1} \neq \lambda$.

Then $g(x, \zeta, p)$ is extended holomorphically to the set

$$
\begin{gathered}
D=\left\{(x, \zeta, p) \in \mathbb{C}^{n} \times \mathbb{C}^{n} \times \mathbb{C} ;|x|<c, \operatorname{Re}\left(\lambda^{-1} \zeta_{1}\right)>a\left|\operatorname{Im}\left(\lambda^{-1} \zeta_{1}\right)\right|,\right. \\
a\left|\zeta_{j}\right|<\left|\zeta_{1}\right| \quad(j=2, \cdots, n),
\end{gathered}
$$$$
\left.\operatorname{Re} p<\varepsilon_{2}|\operatorname{Im} p|, a|p|<|\zeta|\right\}
$$

for some $\varepsilon_{2}>0$, homogeneous with respect to $(\zeta, p)$ of order $(-n)$, and independent of $R$ modulo holomorphic functions at $p=0$.

Moreover, re'hen $G(x, \zeta) \equiv F(x, \zeta)$ modulo rapidly decreasing functions, we have $g(x, \zeta, p) \equiv k(x, \zeta, p)$ modulo holomorphic functions at $p=0$. 
Proof. If $\operatorname{Re} p<0$, the integral (2.1.10) converges absolutely. Deformation of the path of integration proves the first situation. When $G(x, \zeta)$ is rapidly decreasing, it is clear that $g(x, \zeta, p)$ becomes holomorphic at $p=0$. Assume that $G(x, \zeta)=F(x, \zeta)$, then we have

$$
\begin{aligned}
g(x, \zeta, p) & =\frac{1}{2 \pi \sqrt{-1}} \int_{R}^{\infty} \int_{\sigma(\tau \zeta)} k(x, \tau \zeta, q) e^{-q} d q e^{p \tau} \tau^{n-1} d \tau \\
& =\frac{1}{2 \pi \sqrt{-1}} \int_{\sigma(\zeta)} k(x, \zeta, q) \frac{e^{(p-q) R}}{q-p} d q \\
& \equiv k(x, \zeta, p) \text { modulo holomorphic functions at } p=0 .
\end{aligned}
$$

Therefore $\mathcal{E}_{\dot{x}^{*}}^{\boldsymbol{R}}$ is represented as infra-exponential holomorphic functions modulo rapidly decreasing holomorphic functions.

Theorem 2.1.4. Let $\left\{F_{j}\right\}(j=0,1,2, \cdots)$ be a sequence of holomorphic microlocal operators defined in some neighborhood of $\dot{x}^{*}$ $=(0 ; 1,0, \cdots, 0)$.

Assume that the symbol $F_{j}(x, \zeta)$ of $F_{j}$ satisfies the follorving conditions:

(i) $F_{j}(x, \zeta)$ is holomorphic on

$$
\begin{aligned}
& \Gamma=\left\{(x, \zeta) \in C^{n} \times C^{n} ;|x|<c,|\zeta|>r\right. \\
&\left.\operatorname{Re} \zeta_{1}>a\left|\operatorname{Im} \zeta_{1}\right|, a\left|\zeta_{i}\right|<\left|\zeta_{1}\right|(i=2, \cdots, n)\right\}
\end{aligned}
$$

where $0<c \ll 1,1 \ll a$, and $1 \ll r(j=0,1,2, \cdots)$.

(ii) For each compact cone $\Gamma^{\prime} \mathbb{E} \Gamma$, there exists a positive number $A$, and for each positive number $\delta$, there is a positive constant $C_{\hat{o}}$ such that

(2.1.11) $\left|F_{j}(x, \zeta)\right| \leqq C_{\hat{o}} A^{j} j !|\zeta|^{-j} \exp (\delta|\zeta|) \quad$ for $\quad j \geqq 0, \quad(x, \zeta) \in \Gamma^{\prime}$.

Then, there is a sequence $\left\{\psi_{j}(x, \zeta)\right\}(j=0,1, \cdots)$ of holomorphic functions of rapidly decreasing type defined in $\Gamma$ such that $\sum_{j=0}^{\infty}\left(F_{j}(x, \zeta)-\psi_{j}(x, \zeta)\right)$ converges locally uniformly in $\Gamma$ and that the $\operatorname{sum} F(x, \zeta)=\sum_{j=0}^{\infty}\left(F_{j}(x, \zeta)-\psi_{j}(x, \zeta)\right)$ is infra-exponential type in $\Gamma$. That is, the sum $F=\sum_{j=0}^{\infty} F_{j}$ of holomorphic microlocal operators $F_{j}$ exists in $\varepsilon_{\dot{x}^{+}}^{\boldsymbol{R}}$. 
Proof. Take $R>0$ sufficiently large and set

$$
f_{j}(x, \zeta, p)=\frac{1}{(2 \pi \sqrt{-1})^{n}} \int_{(j+1) R}^{+\infty} \zeta_{1}^{-n} F_{j}\left(x, \tau \zeta_{1}^{-1} \zeta\right) \exp \left(\zeta_{1}^{-1} p \tau\right) \tau^{n-1} d \tau
$$

for $j=0,1,2, \cdots$. Because of the homogeneity of $f_{j}(x, \zeta, p)$, we can restrict our discussion about it to $\left\{(x, \zeta, p) ; \zeta_{1}=1\right\}$. Since $F_{j}$ satisfies estimate (2.1.11), we have

$$
\begin{aligned}
& \left|f_{i}(x, \zeta, p)\right| \\
& \quad \leqq(2 \pi)^{-n} C_{\delta} A^{j} j !(j+1)^{n-j} \int_{R}^{\infty} \tau^{n-1-j} \exp ((j+1)(\delta|\zeta|+\operatorname{Re} p) \tau) d \tau \\
& \quad \leqq C_{\hat{o}}^{\prime} B^{j} R^{-j}(j+1)^{-1}\left|(\delta|\zeta|+\operatorname{Re} p)^{-1}\right| \exp ((j+1)(\delta|\zeta|+\operatorname{Re} p) R)
\end{aligned}
$$

if $\delta \mid \zeta_{\mid}+\operatorname{Re} p<0$, here we set $C_{\delta}^{\prime}=3 C_{\hat{\delta}}(2 \pi)^{-n} R^{n-1}, B=3 A$. For each $p$ such that $\operatorname{Re} p<0$, we can take $\delta$ as $\delta|\zeta|+\operatorname{Re} p<0$. Then

$$
\sum_{j=0}^{\infty} C_{\delta}^{\prime} B^{j} R^{-j}(j+1)^{-1}\left|(\delta|\zeta|+\operatorname{Re} p)^{-1}\right| \exp ((j+1)(\delta|\zeta|+\operatorname{Re} p) R)
$$

converges if $R$ is sufficiently large. Hence $\sum_{j=0}^{\infty} f_{j}(x, \zeta, p)=f(x, \zeta, p)$ converges locally uniformly in $\{\operatorname{Re} p<0\}$. Each $f_{j}(x, \zeta, p)$ is extended holomorphically to the set

$$
\begin{gathered}
D=\left\{(x, \zeta, p) \in \mathbb{C}^{n} \times \mathbb{C}^{n} \times \mathbb{C} ;|x|<c, \zeta_{1}=1, a\left|\zeta_{i}\right|<1 \quad(i=2, \cdots, n)\right. \\
\left.\operatorname{Re} p<\varepsilon_{1} \operatorname{Im} p|, a| p|<| \zeta \mid\right\}
\end{gathered}
$$

for some $\varepsilon>0$. Similar argument as above shows $\sum_{j=0}^{\infty} f_{j}(x, \zeta, p)$ converges locally uniformly in $D$. Let $\widetilde{F}_{j}(x, \zeta)$ and $F(x, \zeta)$ be symbols corresponding to $f_{j}(x, \zeta, p)$ and $f(x, \zeta, p)$ respectively. Then,

$$
F_{j}(x, \zeta)-\widehat{F}_{j}(x, \zeta)=\psi_{j}(x, \zeta)
$$

is a holomorphic function of rapidly decreasing type in $\Gamma$ for each $j$ and $\sum_{j=0}^{\infty} F_{j}(x, \zeta)$ converges locally uniformly in $\Gamma$. The sum is equal to $F(x, \zeta)$. Set $F=F\left(x, D_{x}\right)$, then we have

$$
\sum_{j=0}^{\infty} F_{j}\left(x, D_{x}\right)=\sum_{j=0}^{\infty} \widehat{F}_{j}\left(x, D_{x}\right)=F\left(x, D_{x}\right) .
$$

This completes the proof of Theorem 2.1.4. 
Remark 2.1.5. By the method of the proof, we can replace estimate $(2.1 .11)$ in the preceding theorem by

$$
\left|F_{j}(x, \zeta)\right| \leqq C_{\delta} A^{j}(j !)^{s}|\zeta|^{-s j} \exp (\delta|\zeta|) \quad(j=0,1,2, \cdots)
$$

for a positive constant $s$.

Definition 2. 1.6. Let $\dot{x}^{*}=\left(x^{0}, \zeta^{0}\right)$ be a point in $T^{*} X \cong C^{n} \times C^{n}$ and $\Gamma$ be a conic neighborhood of $\dot{x}^{*}$. Let $\left\{F_{j}(x, \zeta)\right\} \quad(j=0,1,2, \cdots)$ be a sequence of holomorphic functions defined in $\Gamma$ such that for each compact cone $\Gamma^{\prime} \mathbb{C} \Gamma$, there is a positive constant $A$ and for each $\delta>0$, there is a posilive number $C_{0}$ such that

$$
\left|F_{j}(x, \zeta)\right| \leqq C_{\delta} A^{j} j !|\zeta|^{-j} \exp (\delta|\zeta|) \quad(j=0,1,2, \cdots)
$$

for $(x, \zeta) \in \Gamma^{\prime}$.

Then we call the formal sum $\sum_{j=0}^{\infty} F_{j}(x, \zeta)$ a formal symbol defined in $\Gamma$. We denote by $E_{\dot{x}^{*}}$ the set of all formal symbols defined in some conic neighborhood of $\dot{x}^{*}$.

By the preceding theorem, the sum $\sum_{j=0}^{\infty} F_{j}\left(x, D_{x}\right)$ defines a holomorphic microlocal operator in $\mathcal{E}_{\dot{x}^{+}}^{\boldsymbol{R}}$ if $\sum_{j=0}^{\infty} F_{j}(x, \zeta)$ belongs to $E_{\dot{x}^{\kappa}} \quad$ (Cf. Boutet de Monvel [1]). The sum is well defined since we have the following theorem.

Theorem 2. 1.7. Let $\sum_{j=0}^{\infty} F_{j}(x, \zeta)$ be a formal symbol defined in a conic neighborhood $\Gamma$ of $\dot{x}^{*}$. Assume that for any compact cone $\Gamma^{\prime} \subset \Gamma$, there is a constant $A>0$ and for each $\delta>0$, there is a number $C_{\hat{0}}>0$ such that

$$
\left|\sum_{j=0}^{N-1} F_{j}(x, \zeta)\right| \leqq C_{\hat{o}} A^{N} N !|\zeta|^{-N} \exp (\delta|\zeta|)
$$

for $(x, \zeta) \in \Gamma^{\prime}, N=1,2, \cdots$.

Then, the sum $\sum_{j=0}^{\infty} F_{j}\left(x, D_{x}\right)$ defines zero operator in $\mathcal{E}_{\dot{x}^{*}}^{\boldsymbol{R}}$

Remark 2.1.8. Let $F\left(x, D_{x}\right)=\sum_{j \in Z} F_{j}\left(x, D_{x}\right)$ be a microdifferential operator $\in \mathcal{E}_{\dot{x}^{*}}^{\infty}$. Here $F_{j}\left(x, D_{x}\right)$ is the homogeneous part of $F$ of order 
$j$ in $D_{x}$. That is, $F_{j}(x, \zeta)$ is a holomorphic function defined in a conic neighborhood $\Gamma$ of $\dot{x}^{*}$ and homogeneous in $\zeta$ of degree $j$ which satisfies the following conditions:

(a) for every $\varepsilon>0$ and every compact cone $\Gamma^{\prime} \mathbb{C} \Gamma$, there is a constant $C>0$ such that

$$
\left|F_{j}(x, \zeta)\right| \leqq \frac{C}{j !} \varepsilon^{j}|\zeta|^{j} \quad \text { for } \quad j \geqq 0, \quad(x, \zeta) \in \Gamma^{\prime}
$$

(b) for every compact cone $\Gamma^{\prime} \mathbb{C} \Gamma$, there is a constant $R>0$ such that

$$
\left|F_{j}(x, \zeta)\right| \leqq(-j) ! R^{-j}|\zeta|^{j} \quad \text { for } \quad j<0, \quad(x, \zeta) \in \Gamma^{\prime} .
$$

Clearly, the symbol of $F_{j}\left(x, D_{x}\right)$ in the sense of Definition 2.1.2 coincides with $F_{j}(x, \zeta)$ (modulo rapidly decreasing functions, of course). Set $F_{+}\left(x, D_{x}\right)=\sum_{j \geq 0} F_{j}\left(x, D_{x}\right)$ and $F_{-}\left(x, D_{x}\right)=\sum_{j<0} F_{j}\left(x, D_{x}\right)$. Condition (a) shows $\sum_{j \geq 0} F_{j}(x, \zeta)$ converges locally uniformly and the sum $\widetilde{F}_{+}(x, \zeta)$ $=\sum_{j \geq 0} F_{j}(x, \zeta)$ satisfies the following:

(a') for every $\varepsilon>0$ and every compact cone $\Gamma^{\prime} \mathbb{C} \Gamma$, there is a constant $C_{\varepsilon}>0$ such that

$$
\left|\widetilde{F}_{+}(x, \zeta)\right| \leqq C_{\varepsilon} \exp (\varepsilon|\zeta|) \quad \text { for } \quad(x, \zeta) \in \Gamma^{\prime} .
$$

Therefore $\widetilde{F}_{+}(x, \zeta)$ is a symbol in the sense of Definition 2.1.2 and $\widetilde{F}_{+}\left(x, D_{x}\right)=F_{+}\left(x, D_{x}\right)$. On the other hand, $\sum_{j<0} F_{j}(x, \zeta)$ does not converge but the formal sum $\sum_{j<0} F_{j}(x, \zeta)$ defines a formal symbol in the sense of Definition 2.1.6. There is a symbol $\widetilde{F}_{-}(x, \zeta)$ such that $\widetilde{F}_{-}(x$, $D)=F_{-}(x, D)$. Thus the symbol of the microdifferetial operator $F(x$, $D)$ in the sense of Definition 2.1 .2 is given by $F_{-}(x, \zeta)+F_{-}(x, \zeta)$. By the way, remark that if we set

$$
\begin{aligned}
& G_{0}(x, \zeta)=F_{0}(x, \zeta), \\
& G_{j}(x, \zeta)=F_{j}(x, \zeta)+F_{-j}(x, \zeta) \text { for } j>0,
\end{aligned}
$$

then the formal sum $\sum_{j \geqq 0} G_{j}(x, \zeta)$ is a formal symbol which defines $F(x, D)$.

2. 2. Next we establish some calculation rules concerning holomorphic microlocal operators, which are expressed in terms of formal symbols. 
Following theorems are direct consequences of $\S 3.3$ in Kataoka [7]. Let $\dot{x}^{*}$ be a point in $T^{*} X \cong \boldsymbol{C}^{n} \times \boldsymbol{C}^{n}$.

Theorem 2.2.1. Let $F=\sum_{j=0}^{\infty} F_{j}\left(x, D_{x}\right)$ be an operator in $\mathcal{E}_{\dot{x}^{*}}^{\boldsymbol{R}}$ where $\sum_{j=0}^{\infty} F_{j}(x, \zeta)$ is a formal symbol. Then the formal adjoint operator $G$ in $\mathcal{E}_{a\left(\dot{x}^{*}\right)}^{\boldsymbol{R}_{1}}\left(a\left(x^{*}\right)=(x,-\zeta)\right.$ for $\left.x^{*}=(x, \zeta) \in T^{*} X\right)$ of $F$ is expressed as follows:

$$
\begin{gathered}
G=\sum_{k=0}^{\infty} G_{k}\left(x, D_{x}\right), \\
G_{k}(x, \zeta)=\sum_{k=|\alpha|+j} \frac{(-1)^{|\alpha|}}{\alpha !}\left(\partial_{\zeta}{ }^{\alpha} \partial_{x}{ }^{\alpha} F_{j}\right)(x,-\zeta), k=0,1,2, \cdots .
\end{gathered}
$$

Here $\sum_{k=0}^{\infty} G_{k}(x, \zeta)$ defines a formal symbol $\in E_{\alpha\left(\dot{x}_{*} *\right.}$.

Theorem 2.2.2. Let $F=\sum_{j=0}^{\infty} F_{j}\left(x, D_{x}\right)$ and $G=\sum_{k=0}^{\infty} G_{k}\left(x, D_{x}\right)$ be holomorphic microlocal operators defined by formal symbols $\sum_{j=0}^{\infty} F_{j}(x$, $\zeta)$ and $\sum_{k=0}^{\infty} G_{k}(x, \zeta)$ in $E_{\dot{x}^{*}}$ respectively. Then the composite operator $R=F G$ is expressed as

$$
R=\sum_{l=0}^{\infty} R_{l}\left(x, D_{x}\right)
$$

Where $\sum_{l=0}^{\infty} R_{l}(x, \zeta)$ is a formal symbol in $E_{\dot{x}^{*}}$ defined by

$$
R_{l}(x, \zeta)=\sum_{l=|\alpha|+j+k} \frac{1}{\alpha !} \partial_{\zeta}^{\alpha} F_{j}(x, \zeta) \cdot \partial_{x}^{\alpha} G_{k}(x, \zeta)
$$

Theorem 2.2.3. Let $x=\left(x_{1}, \cdots, x_{n}\right), y=\left(y_{1}, \cdots, y_{n}\right)$ be the two local coordinate systems of $X$, and $\zeta=\left(\zeta_{1}, \cdots, \zeta_{n}\right), \S=\left(\xi_{1}, \cdots, \xi_{n}\right)$ be the corresponding fiber coordinates of $T^{*} X$. i.e. $\xi_{\nu}=\sum \zeta_{\mu} \partial x_{\mu} / \partial y_{\nu}$. Set

$$
\begin{aligned}
& x=\varphi(y)=\left(\varphi_{1}(y), \cdots, \varphi_{n}(y)\right), \\
& \psi(y)={ }^{t}\left(\left(\partial \varphi_{\mu} / \partial{\nu_{\nu}}_{\nu}\right)_{\mu, \nu}\right)^{-1}, \\
& \Phi_{\mu}(y, v)=\varphi_{\mu}(y)-\varphi_{\mu}\left(y+{ }^{t} \psi(y) \tau^{\prime}\right)+v_{\mu} .
\end{aligned}
$$

Let $F$ be a holomorphic microlocal operator in $\mathcal{E}_{\dot{x}^{*}}^{\boldsymbol{R}}$ Suppose $F=\sum_{j=0}^{\infty} F_{j}\left(x, D_{x}\right)$ for a formal symbol $\sum_{j=0}^{\infty} F_{j}(x, \zeta)$ by the first coordinate system. Then $F$ is expressed by the second coordinate 
system as follows:

$$
\begin{aligned}
& F=\sum_{l=0}^{\infty} \widetilde{F}_{l}\left(y, D_{y}\right), \\
& \quad \widetilde{F}_{l}(y, \xi)=\sum_{\substack{l=|\alpha|=j+k \\
|\beta|+2 j \leq|\alpha|}} \frac{1}{j ! \alpha ! \beta !}\left(\partial_{\xi}^{\alpha} F_{k}\right)(\varphi(y), \psi(y) \xi) \\
& \quad \times\left.\partial_{v}^{\alpha+\beta}\left(\Phi^{\beta}(y, v)\langle\Phi(y, v), \psi(v) \xi\rangle^{j} \operatorname{del}\left(I^{(n)}-\frac{\partial \Phi}{\partial y}(y, v)\right)\right)\right|_{v=0} .
\end{aligned}
$$

Here $\Phi(y, v)=\left(\bar{\Phi}_{1}(y, v), \cdots, \Phi_{n}(y, v)\right), I^{(n)}$ is the identity matrix of size $n$ and $\sum_{l=0}^{\infty} \widetilde{F}_{l}(y, \zeta)$ is a formal symbol.

2.3. In this section, we study the behavior of symbols at $\zeta=\infty$. We use the same notation as in section 1.1: Let $\dot{x}^{*}=(0 ; 1,0, \cdots, 0)$ be a point in $T^{*} X$ and $F$ be an operator in $\mathcal{E}_{\dot{x}^{*}}^{\mathbb{R}}$. Suppose that $k(x, \zeta, p)$ is the normalized Radon transformation of the kernel function of $F$ and that $F(x, \zeta)$ is the symbol of $F$ defined in some conic neighborhood $\Gamma$ of $\dot{x}^{*}$. Then $k(x, \zeta, p)$ is holomorphic on the set $D$ which is defined by $(2.1 .6)$.

Lemma 2.3.1. Let $\rho$ be a number such that $0<\rho<1$. Then the following conditions are equivalent:

(i) For each compact cone $\Gamma^{\prime} \mathbb{C} \Gamma$ there are constants $C_{1}, h_{1}>0$ (resp. For each compact cone $\Gamma^{\prime} \in \Gamma$ and $h_{1}>0$ there is a constant $C_{1}$ ) such that

$$
|F(X, \zeta)| \leqq C_{1} \exp \left(h_{1}|\zeta|^{\rho}\right) \quad \text { for } \quad(x, \zeta) \in \Gamma^{\prime} .
$$

(ii) For each compactly generated cone $K \in D$ there exist constants $C_{2}, h_{2}>0$ (resp. For each compactly generated cone $K \Subset D$ and $h_{2}>0$ there exists a constant $C_{2}$ ) such that

$$
|k(x, \zeta, p)| \leqq C_{2} \exp \left(h_{2}|p|^{-\rho(1-\rho)}\right) \quad \text { for } \quad(x, \zeta, p) \in K \cap\left\{\zeta_{1}=1\right\} .
$$

Proof. (i) $\Rightarrow$ (ii), First case: By Theorem 2.1.3, we have

$$
k(x, \zeta, p)=(2 \pi \sqrt{-1})^{-n} \int_{R}^{\infty} F(x, \tau \zeta) e^{p-} \tau^{n-1} d \tau \quad\left(\zeta_{1}=1\right)
$$

modulo holomorphic functions at $p=0$. For $\operatorname{Re} p \leqq-|\operatorname{Im} p|$, we have 
(2.3.2) $|k(x, \zeta, p)| \leqq(2 \pi)^{-n} \int_{R}^{\infty} C_{1} \exp \left(h_{1}|\zeta|^{\rho} \tau^{\rho}\right) \exp (\operatorname{Re} p \tau) \tau^{n-1} d \tau$

$$
\begin{aligned}
& \leqq C_{1}{ }^{\prime}\left(\frac{2}{|p|}\right)^{n} \sum_{j=0}^{\infty} \frac{(j !)^{\rho}}{j !}\left(2 h_{1}\right)^{j}\left(\frac{4 \rho}{|p|}\right)^{\rho j} \\
& \leqq C_{1}{ }^{\prime} A\left(\frac{2}{|p|}\right)^{n} \exp \left(2(1-\rho)\left(2 h_{1}\left(\frac{4 \rho}{|p|}\right)^{\rho}\right)^{1 /(1-\rho)}\right) \\
& \leqq C_{2} \exp \left(h_{2}|p|^{-\rho(1-\rho)}\right)
\end{aligned}
$$

where $C_{1}{ }^{\prime}, C_{2}, h_{2}$ are suitable positive constants. Here we use the inequality

$$
\sum_{j=0}^{\infty} \frac{x^{j}}{(j !)^{s}} \leqq A \exp \left(2 s x^{1 / s}\right) \quad(s>0, x>0)
$$

for some positive constant $A$.

For $-\operatorname{Im} p \leqq \operatorname{Re} p \leqq \delta \operatorname{Im} p$ or $\operatorname{Im} p \leqq \operatorname{Re} p \leqq-\delta \operatorname{Im} p \quad(0<\delta \ll 1)$, let us deform the path of integration (2.3.1) as $\operatorname{Re} p \tau<0$ respectively. Then the same argument as above shows

$$
|k(x, \zeta, p)| \leqq C_{2} \exp \left(h_{2}|p|^{-\rho /(1-\rho)}\right)
$$

for $-|\operatorname{Im} p| \leqq \operatorname{Re} p \leqq \delta|\operatorname{Im} p|$.

Second case: Suppose that we can find $C_{1}$ for each $h_{1}>0$. Then for each $h_{2}>0$, we can choose $h_{1}, C_{2}$ as the last inequality in (2.3.2) is valid.

(ii) $\Rightarrow$ (i), First case: By the definition (see Theorem 2.1.1),

$$
\begin{aligned}
F(x, s \zeta) & =(2 \pi \sqrt{-1})^{n-1} \int_{s_{0}(s \zeta)}^{s_{1}(s \zeta)} k(x, s \zeta, p) e^{-p} d p \\
& =(2 \pi \sqrt{-1})^{n-1} \int_{s_{0}(\zeta)}^{s_{1}(\zeta)} s^{-n} k(x, \zeta, p) e^{-s p} d p
\end{aligned}
$$

for $s \gg 1$. Take the path $\sigma$ of integration as follows:

$$
\begin{aligned}
& \sigma=\sigma_{0}+\sigma_{\infty}+\sigma_{1}, \\
& \sigma_{i}=\left\{p ; \arg p=\arg s_{i}, \kappa s^{\rho-1}<|p| \leqq\left|s_{i}\right|\right\}, \\
& \sigma_{\infty}=\left\{p ;|p|=\kappa s^{\rho-1}, \arg s_{1} \leqq \arg p \leqq \arg s_{0}\right\}
\end{aligned}
$$

where $i=0,1,0<\kappa \ll 1$.

Then we have 


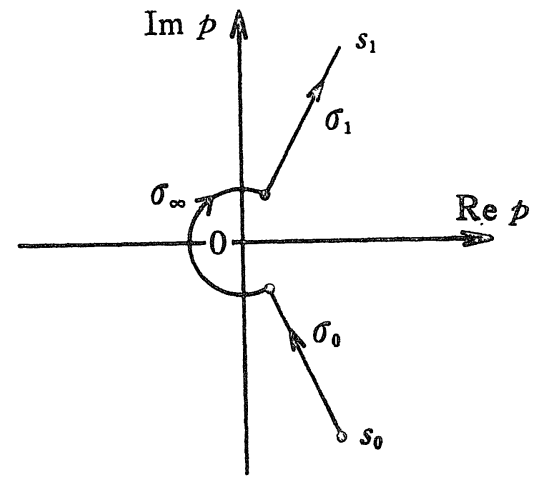

$(2 \cdot 3 \cdot 4) \quad\left|\int_{\sigma_{\infty}} s^{-n} k(x, \zeta, p) e^{-s p} d p\right|$

$$
\begin{aligned}
& \leqq s^{-n} 2 \pi \kappa s^{\rho-1} C_{2} \exp \left(h_{2}\left(\kappa s^{\rho-1}\right)^{-\rho /(1-\rho)}+\kappa s^{\rho}\right) \\
& \leqq C_{2}{ }^{\prime} \exp \left(h_{1} s^{\rho}\right),
\end{aligned}
$$

and

$$
\begin{aligned}
& \left|\int_{\sigma_{i}} s^{-n} k(x, \zeta, p) e^{-s p} d p\right| \\
& \quad \leqq s^{-n} C_{2} \exp \left(h_{2} \kappa s^{\rho}\right) \int_{0}^{\left|s_{i}\right|} \exp \left(-s r \theta_{i}\right) d r \\
& \quad \leqq C_{2}^{\prime} \exp \left(h_{1} s^{\rho}\right)
\end{aligned}
$$

where $C_{2}{ }^{\prime}, h_{1}$ are some constants, $\theta_{i}=\cos \left(\arg s_{i}\right), i=0,1$. Thus we have

$$
|F(x, s \zeta)| \leqq C_{1} \exp \left(h_{1} s^{\rho}\right)
$$

for some constant $C_{1}>0$.

Second case: For each $h_{1}>0$ there exist constants $\kappa, h_{2}, C_{2}^{\prime}$ such that the last inequalities in (2.3.4) and (2.3.5) are valid. Hence for each $h_{1}>0$ there is a constant $C_{1}$ such that

$$
|F(x, s \zeta)| \leqq C_{1} \exp \left(h_{1} s^{n}\right) .
$$

This completes the proof of Lemma 2.3.1.

Definition 2. 3.2. Let $F(x, \zeta)$ be a symbol defined in some conic neighborhood $\Gamma$ of $\dot{x}^{*}$. The holomorphic microlocal operator $F\left(x, D_{x}\right)$ in $\mathcal{E}_{\dot{x}^{*}}^{\mathbb{R}}$ is called of growth order $(\rho)$ (resp. $\{\rho\}$ ) for a number $\rho$ 
$(0 \leqq \rho<1 \quad($ resp. $0<\rho \leqq 1))$ if for each compact cone $\Gamma^{\prime} \Subset \Gamma$ there are constants $C, h>0$ (resp. for each compact cone $\Gamma^{\prime} \subset \Gamma$ and $h>0$ there is a constant $C$ ) such that

$$
|F(x, \zeta)| \leqq C \exp \left(h|\zeta|^{\rho}\right)
$$

for $(x, \zeta) \in \Gamma^{\prime}$.

We denote by $\mathcal{E}_{(\rho), \dot{x}^{*}}^{\boldsymbol{R}}$ (resp. $\left.\mathcal{E}_{\{\rho\}, \dot{x}^{*}}^{\boldsymbol{R}}\right)$ the set of all $F\left(x, D_{x}\right) \in \mathcal{E}_{\dot{x}^{*}}^{\mathbb{R}}$ of growth order at most $(\rho)$ (resp. $\{\rho\})$, and by $\mathcal{E}_{(\rho)}^{\mathbb{R}}$ (resp. $\mathcal{E}_{\{\rho\}}^{\mathbb{R}}$ ) the subsheaf of $\mathcal{E}^{\boldsymbol{R}}$ of germs $\mathcal{E}_{(\rho), \dot{x}^{*}}^{\boldsymbol{R}}$ (resp. $\mathcal{E}_{\{\rho\}, \dot{x}^{*}}^{\boldsymbol{R}}$.

$\mathcal{E}_{\{1\}}^{\mathbb{R}}$ is nothing but $\mathcal{E}^{\boldsymbol{R}}$ and for $0<\rho<1$, we have

$$
\mathcal{E}_{\{\rho\}}^{R} \subsetneq \mathcal{E}_{(\rho)}^{\boldsymbol{R}} \subsetneq \mathcal{E}^{\boldsymbol{R}}
$$

Now we define a subclass of $E_{\dot{x}^{*}}$ corresponding to $\mathcal{E}_{(\rho), \dot{x}^{*}}^{\boldsymbol{R}}$ (resp. $\mathcal{E}_{\{\rho\}, \dot{x}^{*}}^{\boldsymbol{R}}$ ). Let $\rho$ be a number such that $0 \leqq \rho<1$ (resp. $0<\rho \leqq 1)$.

Definition 2.3.3. A formal symbol $\sum_{j=0}^{\infty} F_{j}(x, \zeta)$ defined in $\Gamma$ is called of growth order $(\rho)$ (resp. $\{\rho\})$ if for each compact cone $\Gamma^{\prime} \Subset \Gamma$ there exist positive constants $C, A, h$ (resp. for each compact cone $\Gamma^{\prime} \in \Gamma$ there exists a constant $A$, and for each $h>0$ there is a number C) such that

$$
\left|F_{j}(x, \zeta)\right| \leqq C A^{j} j !|\zeta|^{-j} \exp \left(h|\zeta|^{\rho}\right)
$$

for $j \geqq 0, \quad(x, \zeta) \in \Gamma^{\prime}$.

We denote by $E_{(\rho), \dot{x}^{*}}$ (resp. $E_{\{\rho\}, \dot{x}^{*}}$ ) the set of all formal symbols of growth order at most $(\rho)$ (resp. $\{\rho\}$ ) defined in some conic neighborhood of $\dot{x}^{*}$.

Theorem 2. 3.4. Let $\sum_{j=0}^{\infty} F_{j}(x, \zeta)$ be a formal symbol of growth order $(\rho)$ (resp. $\{\rho\})$. Then the sum $\sum_{j=0}^{\infty} F_{j}\left(x, D_{x}\right)$ is a holomorphic microlocal operator of growth order $(\rho)$ (resp. $\{\rho\})$.

Proof. The case of $(\rho)$. Set

$$
f_{j}(x, \zeta, p)=(2 \pi \sqrt{-1})^{-n} \int_{(j+1) R}^{\infty} F_{j}(x, \tau \zeta) e^{p_{\tau}} \tau^{n-1} d \tau
$$




$$
=\frac{(j+1)^{n}}{(2 \pi \sqrt{-1})^{n}} \int_{R}^{\infty} F_{j}(x,(j+1) \tau \zeta) e^{(j+1) p \tau} \tau^{n-1} d \tau
$$

for $\operatorname{Re} p \leqq-|\operatorname{Im} p|, \zeta_{1}=1$ where $R \gg 1$ is a constant. Suppose that estimate (2.3.6) is valid for $F_{j}(x, \zeta)$. Then, as in the proof of Theorem 2.1.4, one can find $C^{\prime}>0, B>0$ such that

$$
\begin{aligned}
& \left|f_{j}(x, \zeta, p)\right| \\
& \quad \leqq C^{\prime} B^{j} R^{-j} \int_{R}^{\infty} \exp \left(h(j+1)^{\mu} \tau^{\rho}|\zeta|^{\rho}\right) \exp ((j+1) \operatorname{Re} p \tau) d \tau .
\end{aligned}
$$

Moreover, we have

$$
\begin{aligned}
& \left|f_{j}(x, \zeta, p)\right| \\
& \quad \leqq 2 C^{\prime} B^{j} R^{-j} \frac{4}{(j+1)|p|} \sum_{k=0}^{\infty} \frac{(k !)^{\rho}(2 h)^{k}}{k !}\left(\frac{4 \rho}{|p|}\right)^{k} \\
& \quad \leqq C^{\prime} B^{j} R^{-j} \frac{8}{(j+1)|p|} A \exp \left(2(1-\rho)\left(2 h\left(\frac{4 \rho}{|p|}\right)^{\rho}\right)^{1 /(1-\rho)}\right)
\end{aligned}
$$

where $A$ is some constant (see (2.3.3) in the proof of Lemma 2.3.1). Thus we have

$$
\text { (2.3.7) }\left|\sum_{j=0}^{\infty} f_{j}(x, \zeta, p)\right| \leqq C^{\prime \prime} \exp \left(H|p|^{-\rho /(1-\rho)}\right) \quad \text { for } \quad \operatorname{Re} p \leqq-|\operatorname{Im} p|
$$

where $C^{\prime \prime}, H>0$ are suitable constants.

In the same way as in the proof of Lemma 2.3.1, estimate (2.3.7) is obtained for $-|\operatorname{Im} p| \leqq \operatorname{Re} p \leqq \delta|\operatorname{Im} p| \quad(0<\delta \ll 1)$.

Hence $f(x, \zeta, p)=\sum_{j=0}^{\infty} f_{j}(x, \zeta, p)$ satisfies condition (ii) of Lemma 2.3.1, which shows our theorem in the case of $(\rho)$. Another case can be proved in a similar way.

Remark 2.3.5. The preceding theorem is also valid if estimate (2.3.6) is replaced by

$$
\left|F_{j}(x, \zeta)\right| \leqq C A^{j}(j !)^{s}|\zeta|^{-s j} \exp \left(h|\zeta|^{\rho}\right)
$$

for a positive constant $s$.

Corollary 2.3.6. $\mathcal{E}_{(\rho)}^{\mathbb{R}}$ (resp. $\left.\mathcal{E}_{\{\rho\}}^{R}\right)$ is a subring of $\mathcal{E}^{\mathbb{R}}$. 
Corollary 2.3.7. $\mathcal{E}_{(\rho)}^{\boldsymbol{R}} \quad\left(\right.$ resp. $\left.\mathcal{E}_{\{\rho\}}^{\boldsymbol{R}}\right)$ is invariant under taking formal adjoints and coordinate transformations.

\section{§ 3. Invertibility for Holomorphic Microlocal Operators}

3.1. First we investigate invertibility for holomorphic microlocal operators of algebraic growth. Let $\Gamma$ be a conic neighborhood of $\dot{x}^{*} \in T^{*} X \simeq \mathbb{C}^{n} \times \mathbb{C}^{n}$.

Theorem 3.1.1. Let $\sum_{j=0}^{\infty} P_{j}(x, \zeta)$ be a formal symbol defined in $\Gamma$ which satisfies the following conditions: for each compact cone $\Gamma^{\prime} \subset \Gamma$

(i) there exist constants $\lambda \in \boldsymbol{R}, C_{0}>0, C_{1}>0$ such that

$$
C_{0}|\zeta|^{2} \leqq\left|P_{0}(x, \zeta)\right| \leqq C_{1}|\zeta|^{2} \quad \text { for } \quad(x, \zeta) \in \Gamma^{\prime} .
$$

(ii) there exist constants $C_{2}>0, A>0$ such that

$$
\left|P_{j}(x, \zeta)\right| \leqq C_{2} A^{j} j !|\zeta|^{\lambda-j} \text { for } j \geqq 0, \quad(x, \zeta) \in \Gamma^{\prime} .
$$

Then the operator $P\left(x, D_{x}\right)=\sum_{j=0}^{\infty} P_{j}\left(x, D_{x}\right)$ is invertible in the ring $\mathcal{E}^{\boldsymbol{R}}$.

Proof. First we construct the right inverse of $P\left(x, D_{x}\right)$. Set

$$
\begin{aligned}
& Q_{0}(x, \zeta)=1 / P_{0}(x, \zeta), \\
& Q_{l}(x, \zeta)=-\sum_{\substack{l=|\alpha|+j+k \\
k<l}} \frac{1}{\alpha !} \partial_{\zeta}^{\alpha} P_{j}(x, \zeta) \cdot \partial_{x}^{\alpha} Q_{k}(x, \zeta) / P_{0}(x, \zeta) \\
& \text { for } l \geqq 1 .
\end{aligned}
$$

If $\sum_{l=0}^{\infty} Q_{l}(x, \zeta)$ is a formal symbol, it is clear that $P\left(x, D_{x}\right) Q\left(x, D_{x}\right)=1$ for $Q\left(x, D_{x}\right)=\sum_{l=0}^{\infty} Q_{l}\left(x, D_{x}\right)$.

Let us prove that $\sum_{l=0}^{\infty} Q_{l}(x, \zeta)$ is a formal symbol defined in $\Gamma$. Note that each $Q_{l}(x, \zeta)$ is holomorphic in $\Gamma$ by the definition. For each compact cone $\Gamma^{\prime} \Subset \Gamma$ one can find $\delta_{2}>0$ by means of Cauchy's inequality such that

$$
\left|\partial_{\zeta}{ }^{\alpha} P_{j}(x, \zeta)\right| \leqq C_{2} A^{j} \alpha ! j !|\zeta|^{\lambda-j-|\alpha|} \delta_{2}^{-|\alpha|}
$$

for $j \geqq 0,(x, \zeta) \in \Gamma^{\prime}, \alpha \in \mathbb{Z}_{+}{ }^{n}$. 
We prove the following inequality by induction; for each compact cone $\Gamma^{\prime} \Subset \Gamma$ there exist constants $C_{3}, B, \delta_{1}, \delta_{2}$ and $M>1$ such that

$$
\left|Q_{k}(x, \zeta)\right| \leqq C_{3} B^{k} k ! \delta_{1}^{-M k} \delta_{2}^{-k}|\zeta|^{-\lambda-k}
$$

for $k \geqq 0, \quad(x, \zeta) \in \Gamma^{\prime}$.

Set $C_{3}=C_{0}{ }^{-1}$, then the inequality is valid for $k=0$.

Assume the inequality for each $k$ such that $k<l$. Then we have

$$
\left|\partial_{x}^{\alpha} Q_{k}(x, \zeta)\right| \leqq C_{3} B^{k}(|\alpha|+k) ! \delta_{1}^{-M(|\alpha|+k)} \delta_{2}^{-k}|\zeta|^{-\lambda-k}
$$

for $(x, \zeta) \in \Gamma^{\prime}, \alpha \in Z_{+}{ }^{n}, k<l$. Here $\delta_{1}$ (resp. $\delta_{2}$ ) is the distance from $\pi\left(\Gamma^{\prime}\right)$ to $\partial \pi(\Gamma)$ (resp. from the fiber of $\gamma_{\boldsymbol{R}}\left(\Gamma^{\prime}\right)$ to the fiber of $\partial \gamma_{\mathbb{R}}(\Gamma)$ where $\gamma_{\boldsymbol{R}}$ is the projection $\left.T^{*} X-X \rightarrow S^{*} X\right)$. Thus we have

$$
\begin{aligned}
& \left|Q_{l}(x, \zeta)\right| \\
& \quad \leqq \sum_{\substack{l=|\alpha|+j+k \\
k<l}} C_{2} A^{j} j !|\zeta|^{-j-i \alpha \mid} \delta_{2}{ }^{-|\alpha|} C_{3}{ }^{2} B^{k}(|\alpha|+k) ! \delta_{1}{ }^{-M(|\alpha|+k)} \delta_{2}^{-k}|\zeta|^{-\lambda-k} \\
& \quad \leqq C_{3} B^{l} l ! \delta_{1}^{-M l} \delta_{2}^{-l}|\zeta|^{-\lambda-l} C_{2} C_{3} \sum_{\substack{l=\nu+j+k \\
k<l}} n^{\prime \prime} A^{j} B^{-\nu-j} \delta_{1}^{M f j} \delta_{2}{ }^{j}
\end{aligned}
$$

Since $\delta_{1}, \delta_{2}$ are sufficiently small, there exists $B$ independently of $l$ such that

$$
C_{2} C_{3} \sum_{\substack{l=\nu+j+k \\ k<l}} n^{\nu} A^{j} B^{-\nu-j} \delta_{1}^{M j} \delta_{2}^{j} \leqq 1
$$

So we have

$$
\left|Q_{l}(x, \zeta)\right| \leqq C_{3} B^{l} l ! \delta_{1}^{-\lambda l} \delta_{2}^{-l}|\zeta|^{-\lambda-l} \quad \text { for } \quad l \geqq 0, \quad(x, \zeta) \in \Gamma^{\prime} .
$$

Hence $\sum_{k=0}^{\infty} Q_{k}(x, \zeta)$ is a formal symbol defined in $\Gamma$ and $Q\left(x, D_{x}\right)$ $=\sum_{k=0}^{\infty} Q_{k}\left(x, D_{x}\right)$ is the right inverse of $P\left(x, D_{x}\right)$.

It is clear from the method of the proof given above that we can construct the left inverse $S$ of $P$ also. Then we have $S=S(P Q)=(S P) Q$ $=Q$, hence $S=Q$ holds.

3. 2. Next we study invertibility for holomorphic microlocal operators with constant coefficients.

Definition 3.2.1. A holomorphic microlocal operator $P$ defined in an open set of $T^{*} C^{n}$ is said to be with constant coefficients if 


$$
\left[D_{j}, P\right]=D_{j} P-P D_{j}=0 \quad \text { for } \quad j=1,2, \cdots, n
$$

where $D_{j}=\partial / \partial x_{j}$.

The following theorem is trivial.

Theorem 3.2.2. Let $P$ be a holomorphic microlocal operator with constant coefficients defined in a neighborhood of $\dot{x}^{*} \in T^{*} C^{n}$. Let $P(x, \zeta)$ be the symbol of $P$ defined in a conic neighborhood $\Gamma$ of $\dot{x}^{*}$. Assume that for each compact cone $\Gamma^{\prime} \subset \Gamma$ and $\varepsilon>0$ there exists $C_{\varepsilon}>0$ such that

$$
|P(x, \zeta)| \geqq C_{\varepsilon} \exp (-\varepsilon|\zeta|) \quad \text { for } \quad(x, \zeta) \in \Gamma^{\prime} .
$$

Then $P=P\left(x, D_{x}\right)$ is invertible in $\mathcal{E}_{\dot{x}^{*}}^{\boldsymbol{R}}$,

3.3. Now we prove the following theorem which gives a sufficient condition of invertibility for holomorphic microlocal operators in a rather wide class which includes (micro) differential operators of infinite order with variable coefficients (of growth order at most (1/2)). Let $\Gamma$ be a conic neighborhood of $\dot{x}^{*} \in T^{*} X$.

Theorem 3. 3.1. Let $P(x, \zeta)$ be a symbol defined in $\Gamma$. Let $\rho$ be a number such that $0 \leqq \rho \leqq 1 / 2$. Assume that there exist positive constants $h, C_{0}, C_{1}$ such that

$$
C_{0} \exp \left(-h|\zeta|^{\rho}\right) \leqq|P(x, \zeta)| \leqq C_{1} \exp \left(h|\zeta|^{\rho}\right) \quad \text { for } \quad(x, \zeta) \in \Gamma .
$$

Then the holomorphic microlocal operator $P=P\left(x, D_{x}\right)$ is invertible in the ring $\mathcal{E}_{\dot{x}^{*}}^{\boldsymbol{R}}$. The inverse $U$ of $P$ is given by the form $U=Q R$. Here $Q$ is a holomorphic microlocal operator with symbol $(P(x, \zeta))^{-1}$ and $R$ is an operator of order 0. The principal part of $R$ is

$$
\exp \left(\sum_{j=1}^{n} \partial_{\zeta_{j}} p(x, \zeta) \cdot \partial_{x_{j}} p(x, \zeta)\right)
$$

where $p(x, \zeta)=\log P(x, \zeta)$.

Lemma 3.3.2. Let $P(x, \zeta)$ be a symbol defined in $\Gamma$. Let $\mu$ be a number such that $0 \leqq \rho<1 \quad$ (resp. $0<\rho \leqq 1)$. Suppose that there 
exist positive constants $h, C_{0}, C_{1}$ (resp. for each $h>0$ there exist positive constants $C_{0}, C_{1}$ ) such that

(3. 3. 1) $\quad C_{0} \exp \left(-h|\zeta|^{\rho}\right) \leqq|P(x, \zeta)| \leqq C_{1} \exp \left(h|\zeta|^{\rho}\right) \quad$ for $\quad(x, \zeta) \in \Gamma$.

Then there are holomorphic functions $R_{\nu}^{(\alpha)}(x, \zeta), S_{\mu}^{(\alpha)}(x, \zeta)$ defined in $\Gamma$ for all $\alpha \in \mathbb{Z}_{+}^{n}, \nu, \mu=0,1, \cdots,|\alpha|-1$ satisfying

(a) for each $\alpha(|\alpha| \geqq 1)$

$$
\begin{aligned}
& \left(a_{1}\right) \quad \partial_{\zeta}{ }^{\alpha} P(x, \zeta) / P(x, \zeta)=\sum_{\nu=0}^{|\alpha|-1} R_{\nu}^{(\alpha)}(x, \zeta) \\
& \left(a_{2}\right) \quad \partial_{x}{ }^{\alpha} P(x, \zeta) / P(x, \zeta)=\sum_{\mu=0}^{|\alpha|-1} S_{\mu}^{(\alpha)}(x, \zeta)
\end{aligned}
$$

(b) for each compact cone $\Gamma^{\prime} \Subset \Gamma$ there exist positive constants $C, \delta_{1}, \delta_{2}, H, A$ and $M \geqq 2$ (resp. for each compact cone $\Gamma^{\prime} \mathbb{C} \Gamma$ there exist positive constants $C, \delta_{1}, \delta_{2}, M(\geqq 2)$ and for each $H>0$ there exists $A>0$ ) such that

$\left(b_{1}\right) \quad\left|R_{\nu}^{(\alpha)}(x, \zeta)\right| \leqq \frac{(|\alpha|-1) !}{(|\alpha|-\nu-1) !} C^{|\alpha|-\nu-1} \delta_{2}^{-M|\alpha|}\left(H|\zeta|^{\rho}+A\right)^{|\alpha|-\nu|\zeta|^{-|\alpha|}}$

$\left(b_{2}\right) \quad\left|S_{\mu}^{(a)}(x, \zeta)\right| \leqq \frac{(|\alpha|-1) !}{(|\alpha|-\mu-1) !} C^{|\alpha|-\mu-1} \delta_{1}^{-M|\alpha|}\left(H|\zeta|^{\rho}+A\right)^{|\alpha|-\mu}$

for $\nu, \mu=0,1, \cdots,|\alpha|-1, \quad(x, \zeta) \in \Gamma^{\prime}$.

Proof of Lemma 3.3.2. Let $p(x, \zeta)$ be a holomorphic function defined in $\Gamma$ such that $P(x, \zeta)=\exp (p(x, \zeta))$. Then we have

$$
\begin{aligned}
\partial_{\zeta}{ }^{\alpha} P(x, \zeta) & / P(x, \zeta)=\exp (-p(x, \zeta)) \partial_{\zeta}{ }^{a} \exp (p(x, \zeta)) \\
= & \left(D_{\zeta_{1}}+\partial_{\zeta_{1}} p(x, \zeta)\right)^{\alpha_{1}} \ldots\left(D_{\zeta_{n}}+\partial_{\zeta_{n}} p(x, \zeta)\right)^{\alpha_{n}} 1 \\
= & \left(D_{\zeta}+\operatorname{grad}_{\zeta} p(x, \zeta)\right)^{a} 1 .
\end{aligned}
$$

Here the last term is the homogeneous part of order 0 of the differential operator $\left(D_{\zeta}+\operatorname{grad}_{\zeta} p(x, \zeta)\right)^{\alpha}$.

Set

$$
R_{0}^{(\alpha)}(x, \zeta)=\partial_{\zeta}{ }^{\alpha} p(x, \zeta)=\partial_{\zeta}{ }^{\alpha} \log P(x, \zeta)
$$

for $|\alpha|=1$. Suppose that $R_{\nu}^{(\alpha)}$ is defined for each $\alpha$ such that $|\alpha| \leqq l-1$ $(l \geqq 2)$, then we define $R_{\nu}^{(\alpha)}$ for $|\alpha|=l$ as follows: If $\alpha=(0, \cdots, 0, \alpha$, $\left.\alpha_{j+1}, \cdots, \alpha_{n}\right)$ with $\alpha_{j} \neq 0$, put $\beta=\left(0, \cdots, 0, \alpha_{j}-1, \alpha_{j+1}, \cdots, \alpha_{n}\right)$ and set 
(3. 3. 2. 1) $\quad R_{l-1}^{(\alpha)}(x, \zeta)=\partial_{\zeta_{j}} R_{l-2}^{(\beta)}(x, \zeta)$,

(3. 3. 2. 2) $\quad R_{\nu}^{(\alpha)}(x, \zeta)=\partial_{\zeta,} R_{\nu-1}^{(\beta)}(x, \zeta)+\partial_{\zeta_{j}} p(x, \zeta) \cdot R_{\nu}^{(\beta)}(x, \zeta)$

$$
\text { for } 1 \leqq \nu \leqq l-2 \text {, }
$$

(3.3.2.3) $\quad R_{0}^{(\alpha)}(x, \zeta)=\partial_{\zeta_{j}} p(x, \zeta) \cdot R_{0}^{(\beta)}(x, \zeta)$.

Then we have $\left(a_{1}\right)$. Indeed,

$$
\begin{aligned}
\partial_{\zeta}{ }^{\alpha} P(x, \zeta) / P(x, \zeta) & \\
= & \left(D_{\zeta_{j}}+\partial_{\zeta_{j}} p(x, \zeta)\right)\left(\partial_{\zeta}{ }^{\beta} P(x, \zeta) / P(x, \zeta)\right) \\
= & \left(D_{\zeta_{j}}+\partial_{\zeta_{j}} p(x, \zeta)\right) \sum_{\nu=0}^{l-2} R_{\nu}^{(\beta)}(x, \zeta) \\
= & \sum_{\nu=0}^{l-2} \partial_{\zeta_{j}} R_{\nu}^{(\beta)}(x, \zeta)+\sum_{\nu=0}^{l-2} \partial_{\zeta_{j}} p(x, \zeta) \cdot R_{\nu}^{(\beta)}(x, \zeta) \\
= & \partial_{\zeta_{j}} R_{l-2}^{(\beta)}(x, \zeta)+\sum_{\nu=1}^{l-2}\left(\partial_{\zeta_{j}} R_{\nu-1}^{(\beta)}(x, \zeta)+\partial_{\zeta_{j}} p(x, \zeta) \cdot R_{\nu}^{(\beta)}(x, \zeta)\right) \\
& \quad+\partial_{\zeta_{j}} p(x, \zeta) \cdot R_{0}^{(\beta)}(x, \zeta) .
\end{aligned}
$$

Let us prove the estimate for $R_{\nu}^{(\alpha)}(x, \zeta)$ by induction. Note that

Sublemma. Let $f(z)$ be a holomorphic function of one variable $z$ which is holomorphic in $\left|z-z_{0}\right|<r\left(z_{0} \in C, r>0\right)$, continuous and non zero in $\left|z-z_{0}\right| \leqq r$. Then we have

$$
\frac{f^{\prime}(z)}{f(z)}=\frac{1}{\pi} \int_{0}^{2 \pi} \log \left|f\left(z_{0}+r e^{i \theta}\right)\right| \frac{z_{0}+r e^{i \theta}}{\left(z_{0}+r e^{i \theta}-z\right)^{2}} d \theta
$$

for $\left|z-z_{0}\right|<r \quad(i=\sqrt{-1})$.

Proof of Sublemma. Apply the well known formula in the theory of functions of one complex variable

$$
\varphi(z)=i \operatorname{Im} \varphi\left(z_{0}\right)+\frac{1}{2 \pi} \int_{0}^{2 \pi} \operatorname{Re} \varphi\left(z_{0}+r e^{i \theta}\right) \frac{z_{0}+r e^{i \theta}+z}{z_{0}+r e^{i \theta}-z} d \theta
$$

to $\varphi(z)=\log f(z)$ and operate $\frac{d}{d z}$ on the both members.

Proof of Lemma 3.3.2 continued. The estimate for $R_{0}^{(\alpha)}(x, \zeta)$ for $|\alpha|=1$ is obtained by means of Sublemma; for $\alpha=(1,0, \cdots, 0)$, we have 


$$
\left|\partial_{\zeta_{1}} p(x, \zeta)\right| \leqq \frac{1}{\pi} \int_{0}^{2 \pi}|\log | P\left(x, \zeta_{1}+r e^{i \theta}, \zeta^{\prime}\right)|| \frac{\left|\zeta_{1}+r e^{i \theta}\right|}{r^{2}} d \theta
$$

where $\zeta=\left(\zeta_{1}, \zeta^{\prime}\right)$. If $(x, \zeta) \in \Gamma^{\prime} \subset \subset$, one can put $r=\delta_{2}|\zeta|$ for some $\delta_{2}>0$. In view of $(3.3 .1)$ there exist positive numbers $H, A$ (resp. for each $H>0$ there exists $A>0$ ) such that

$$
\left|\partial_{\zeta_{1}} p(x, \zeta)\right| \leqq\left(H|\zeta|^{\rho}+A\right)|\zeta|^{-1} \delta_{2}^{-M}
$$

where $M$ is a constant $\geqq 2$.

Suppose estimate $\left(b_{1}\right)$ for all $\alpha$ such that $|\alpha| \leqq l-1(l \geqq 2)$. Then for each $\alpha$ such that $|\alpha|=l$, we have from (3.3.2.2) that

$$
\begin{aligned}
& \left|R_{\nu}^{(\alpha)}(x, \zeta)\right| \\
& \leqq\left|\partial_{\zeta_{j}} R_{\nu-1}^{(\beta)}(x, \zeta)\right|+\left|\partial_{\zeta_{j}} p(x, \zeta)\right| \cdot\left|R_{\nu}^{(\beta)}(x, \zeta)\right| \\
& \leqq \frac{(|\beta|-1) !}{(|\beta|-\nu) !} C^{|\beta|-\nu}(1-1 /|\beta|)^{-M|\beta|} \delta_{2}^{-M|\beta|} \\
& \quad \times\left(H|\zeta|^{\rho}+A\right)^{|\beta|-\nu+1}|\zeta|^{-|\beta|}\left(\delta_{2}|\zeta| /|\beta|\right)^{-1} \\
& \quad+\frac{(|\beta|-1) !}{(|\beta|-\nu-1) !} C^{|\beta|-\nu-1} \delta_{2}{ }^{-M l}\left(H|\zeta|^{\rho}+A\right)^{|\beta|-\nu}|\zeta|^{-|\beta|} \\
& \quad \times\left(H|\zeta|^{\rho}+A\right)|\zeta|^{-1} \delta_{2}{ }^{-M I} \\
& \leqq \frac{(l-1) !}{(l-\nu-1) !} C^{l-\nu-1} \delta_{2}^{-M l}\left(H|\zeta|^{\rho}+A\right)^{l-\nu}|\zeta|^{-l}\left(\delta_{2}{ }^{M-1} e^{M}+\frac{l-\nu-1}{l-1} C^{-1}\right)
\end{aligned}
$$

for $1 \leqq \nu \leqq l-2$. Since $M>1$, and $\delta_{2}$ is sufficiently small, one can choose $C$ independently of $l, \nu$ such that

$$
\delta_{2}^{M-1} e^{M}+\frac{l-\nu-1}{l-1} C^{-1} \leqq 1
$$

Therefore we have $\left(b_{1}\right)$ for $1 \leqq \nu \leqq l-2$. By the definition, it is clear that

$$
\begin{aligned}
& R_{0}^{(\alpha)}(x, \zeta)=\left(\operatorname{grad}_{\zeta} p(x, \zeta)\right)^{\alpha}, \\
& R_{l-1}^{(\alpha)}(x, \zeta)=\partial_{\zeta}{ }^{\alpha} p(x, \zeta) .
\end{aligned}
$$

Thus $\left(b_{1}\right)$ is also valid for $\nu=0$ and $\nu=l-1$. This completes the proof of the estimate for $R_{\nu}^{(\alpha)}(x, \zeta)$. In a similar way, one can define $S_{\mu l}^{(\alpha)}(x, \zeta)$ and have $\left(b_{2}\right)$. 
Proof of Theorem 3.3. 1. Put $Q(x, \zeta)=1 / P(x, \zeta)$, then we have

$$
|Q(x, \zeta)| \leqq C_{0}^{-1} \exp \left(h|\zeta|^{\rho}\right) \quad \text { for } \quad(x, \zeta) \in \Gamma
$$

and thus $Q(x, \zeta)$ is a symbol defined in $\Gamma$. Let $Q\left(x, D_{x}\right)$ be the holomorphic microlocal operator defined by the symbol $Q(x, \zeta)$. Consider the composite operator $L=P\left(x, D_{x}\right) Q\left(x, D_{x}\right)$ of $P\left(x, D_{x}\right)$ and $Q\left(x, D_{x}\right)$. Then $L$ is expressed as (see Theorem 2.2.2)

$$
L=\sum_{i=0}^{\infty} L_{l}\left(x, D_{x}\right)
$$

where $\sum_{l=0}^{\infty} L_{l}(x, \zeta)$ is a formal symbol defined by

$$
L_{l}(x, \zeta)=\sum_{|\alpha|=l} \frac{1}{\alpha !} \partial_{\zeta}{ }^{\alpha} P(x, \zeta) \cdot \partial_{x}{ }^{\alpha} Q(x, \zeta) \quad \text { for } l \geqq 0
$$

Apply Lemma 3.3 .2 to $P(x, \zeta)$ and $Q(x, \zeta)$ respectively. Then one can find $R_{\nu}^{(\alpha)}(x, \zeta), S_{\mu}^{(\alpha)}(x, \zeta)$ for $\alpha \in Z_{+}^{n}(|\alpha| \geqq 1), \nu, \mu=0,1, \cdots$, $|\alpha|-1$ such that

$$
\begin{aligned}
& \frac{\partial_{\zeta}{ }^{\alpha} P(x, \zeta)}{P(x, \zeta)}=\sum_{\nu=0}^{|\alpha|-1} R_{\nu}^{(\alpha)}(x, \zeta), \\
& \frac{\partial_{x}^{\alpha} Q(x, \zeta)}{Q(x, \zeta)}=\sum_{\mu=0}^{|\alpha|-1} S_{\mu}^{(\alpha)}(x, \zeta)
\end{aligned}
$$

and that estimates (b) in the lemma is valid.

It is clear that $L_{0}(x, \zeta)=1$. When $l \geqq 1, L_{l}(x, \zeta)$ can be written as

$$
\begin{aligned}
L_{l}(x, \zeta) & =\sum_{|\alpha|=l} \frac{1}{\alpha !} \cdot \frac{\partial_{\zeta}^{\alpha} P(x, \zeta)}{P(x, \zeta)} \cdot \frac{\partial_{x}^{\alpha} Q(x, \zeta)}{Q(x, \zeta)} \\
& =\sum_{|\alpha|=l} \frac{1}{\alpha !} \sum_{k=0}^{2 l-2} \sum_{\substack{\nu+\mu=k \\
0 \leq \nu \leq l-1 \\
0 \leq \mu \leq l-1}} R_{\nu}^{(\alpha)}(x, \zeta) S_{\mu}^{(\alpha)}(x, \zeta) .
\end{aligned}
$$

Observe that $R_{\nu}^{(\alpha)}(x, \zeta) S_{\mu}^{(\alpha)}(x, \zeta)$ satisfies the following estimate in virtue of $(b)$ in Lemma 3.3.2 $(|\alpha|=l, \nu+\mu=k)$ :

(3. 3. 3) $\left|R_{\nu}^{(\alpha)}(x, \zeta) S_{\mu}^{(\alpha)}(x, \zeta)\right|$

$$
\begin{aligned}
& \leqq \frac{(l-1) !^{2}}{(l-\nu-1) !(l-\mu-1) !}\left(\delta_{1} \delta_{2}\right)^{-M l} C^{2 l-\nu-\mu-2}\left(H|\zeta|^{\rho}+A\right)^{2 l-\nu-\mu}|\zeta|^{-l} \\
& \leqq 4^{l-1} k ! \delta^{-l} C^{2 l-k-2}\left(H|\zeta|^{\rho-1 / 2}+A|\zeta|^{-1 / 2}\right)^{2 l}\left(H|\zeta|^{\rho}+A\right)^{-k}
\end{aligned}
$$


for $(x, \zeta) \in \Gamma^{\prime} \subset \Gamma$. Here we set $\delta=\left(\delta_{1} \delta_{2}\right)^{M}$.

Put

$$
K_{k}(x, \zeta)=\sum_{l \geq(k+3) / 2} \sum_{|\alpha|=l} \frac{1}{\alpha !} \sum_{\nu+\mu=k} R_{\nu}^{(a)}(x, \zeta) S_{\mu}^{(\alpha)}(x, \zeta)
$$

then the sum converges locally uniformly in $I$. Indeed, in view of (3.3.3), we have

(3.3.4) $\left|K_{k}(x, \zeta)\right|$

$$
\begin{aligned}
& \leqq \sum_{l \geq \frac{k+3}{2}} \sum_{|a|=l} \frac{1}{\alpha !}(k+1) ! 4^{l-1} \delta^{-l} C^{2 l-k-2}\left(H|\zeta|^{\rho-1 / 2}+A|\zeta|^{-1 / 2}\right)^{2 l} \\
& \times\left(H|\zeta|^{\rho}+A\right)^{-k} \\
& \leqq C^{-2-k}(k+1) !\left(H \mid \zeta !^{\mid \rho}+A\right)^{-k} 4^{-1} \sum_{l \sum^{\frac{k+3}{2}}} \frac{\left(4 n C^{2} \delta^{-1}\right)^{l}}{l !} \\
& \times\left(H|\zeta|^{\rho-1 / 2}+A|\zeta|^{\rho-1 / 2}\right)^{2 l} \\
& \leqq\left\{\begin{array}{c}
2^{2 m+1}{C_{2}}^{m+1} m !|\zeta|^{-m-1}\left(H|\zeta|^{\rho}+A\right)^{2} \\
\times \exp \left(C_{2}\left(H|\zeta|^{\rho-1 / 2}+A|\zeta|^{-1 / 2}\right)^{2}\right) \\
\text { for } \quad k=2 m \quad(m=0,1,2, \cdots), \\
2^{2 m+2} C_{2}{ }^{m+2} m !|\zeta|^{-m-2}\left(H|\zeta|^{\rho}+A\right)^{3} \\
\times \exp \left(C_{2}\left(H|\zeta|^{\rho-1 / 2}+A|\zeta|^{-1 / 2}\right)^{2}\right) \\
\text { for } k=2 m+1 \quad(m=0,1,2, \cdots)
\end{array}\right.
\end{aligned}
$$

in $\Gamma^{\prime}$. Here we set $C_{2}=4 n C^{2} \delta^{-1}$. Because $\rho \leqq 1 / 2$, there is a constant $C_{3}>0$ such that

$$
\exp \left(C_{2}\left(H|\zeta|^{\rho-1 / 2}+A|\zeta|^{-1 / 2}\right)^{2}\right) \leqq C_{3} \quad \text { for } \quad(x, \zeta) \in \Gamma^{\prime} .
$$

Since

$$
1+K_{0}(x, \zeta)=\exp \left(-\sum_{j=1}^{n} \partial_{\zeta_{j}} p(x, \zeta) \cdot \partial_{x_{j}} p(x, \zeta)\right),
$$

there exist constants $C_{4}>0, C_{5}>0$ such that

$$
C_{4} \leqq\left|1+K_{0}(x, \zeta)+K_{1}(x, \zeta)\right| \leqq C_{5} \quad \text { for } \quad(x, \zeta) \in \Gamma^{\prime} .
$$

Therefore if one sets

$$
\begin{aligned}
& \widetilde{K}_{0}(x, \zeta)=1+K_{0}(x, \zeta)+K_{1}(x, \zeta), \\
& \widetilde{K}_{m}(x, \zeta)=K_{2 m}(x, \zeta)+K_{2 m+1}(x, \zeta) \quad \text { for } \quad m \geqq 1
\end{aligned}
$$


then $\sum_{m=0}^{\infty} \widetilde{K}_{m}(x, \zeta)$ is a formal symbol which satisfies the condition of Theorem 3.1.1. On the other hand, we have

$$
\begin{aligned}
\sum_{m=0}^{\infty} \widetilde{K}_{m}\left(x, D_{x}\right) & =1+\sum_{k=0}^{\infty} K_{k}\left(x, D_{x}\right) \\
& =\sum_{l=0}^{\infty} L_{1}\left(x, D_{x}\right) \\
& =L\left(x, D_{x}\right) .
\end{aligned}
$$

Thus $L\left(x, D_{x}\right)$ has the inverse $\left(L\left(x, D_{x}\right)\right)^{-1}$ in $\mathcal{E}_{\dot{x}^{*}}^{\boldsymbol{R}}$

Now set $U=Q\left(x, D_{x}\right)\left(L\left(x, D_{x}\right)\right)^{-1}$, then $U$ is the right inverse of $P\left(x, D_{x}\right)$.

The construction of the left inverse $V$ of $P\left(x, D_{x}\right)$ is left to the reader. It is easy to see $U=V$. Hence $P\left(x, D_{x}\right)$ is invertible in $\mathcal{E}_{\dot{x}^{*}}^{\boldsymbol{R}}$.

At the same time, we obtain the following theorem which was suggested by Kataoka (see (3.3.4) and Theorem 2.3.4).

Theorem 3. 3. 3. Let $\rho$ be a number such that $1 / 2 \leqq \rho<1$. Let $P(x, \zeta)$ be a symbol of growth order $(\rho)$ defined in $\Gamma$. Assume that there exist positive constants $h, C_{0}, C_{1}$ such that

$$
C_{0} \exp \left(-h|\zeta|^{\rho}\right) \leqq|P(x, \zeta)| \leqq C_{1} \exp \left(h|\zeta|^{\rho}\right) \quad \text { for } \quad(x, \zeta) \in \Gamma .
$$

Set $Q(x, \zeta)=1 / P(x, \zeta)$. Then the composite operators $P\left(x, D_{x}\right) Q(x$, $\left.D_{x}\right)$ and $Q\left(x, D_{x}\right) P\left(x, D_{x}\right)$ are of growth order at most $(2 \rho-1)$ at $\dot{x}^{*}$.

3. 4. Let $M$ be a real analytic manifold, $X$ a complexification of $M$, and $T_{M}^{*} X$ the conormal bundle of $M$ in $T^{*} X$. We denote by $\mathscr{C}_{M}$ the sheaf on $T_{M}^{*} X$ of microfunctions. Since $\mathscr{C}_{M}$ is a left $\left.\mathcal{E}_{X}^{\boldsymbol{R}}\right|_{T_{M}^{*} X^{*}}$-module, Theorem 3.3.1 implies the following theorem.

Theorem 3. 4. 1. Let $\dot{x}^{*}$ be a point in $T_{M}^{*} X$ and $P$ a holomorphic microlocal operator defined in a neighborhood of $\dot{x}^{*}$. Assume that the symbol $P(x, \zeta)$ of $P$ (by a local coordinate system) satisfies the condition of Theorem 3.3.2. Then 


$$
P: \mathscr{C}_{M} \rightarrow \mathscr{C}_{M}
$$

is bijective at $\dot{x}^{*}$.

\section{Acknowledgement}

The author wishes to express his gratitude to Professor H. Komatsu for the guidance and encouragement. He also wishes to express his thanks to Dr. K. Kataoka for valuable discussion.

\section{References}

[1] L. Boutet de Monvel, Opérateurs pseudo-différentiels analytiques et opérateurs d'ordre infini, Ann. Inst. Fourier, Grenoble, 22 (1972), 229-268.

[2] M. Kashiwara and T. Kawai, On holonomic systems of microdifferential equations III, Publ. RIMS, Kyoto Univ., 17 (1981), 813-979.

[3] — Second-microlocalization and asymptotic expansions, Lect. Notes in Phys. No. 126, Springer, pp. 21-76 (1980).

[4] M. Kashiwara and P. Schapira, Problème de Cauchy pour les systèmes microdifférentiels dans le domaine complexe, Inventiones math. 46 (1978), 17-38.

[5] - Micro-hyperbolic systems, Acta Math. 142 (1979), 1-55.

[6] K. Kataoka, On the theory of Radon transformations of hyperfunctions, Master's thesis in the Univ. of Tokyo (1976, in Japanese).

[7] - On the theory of Radon transformations of hyperfunctions, J. Fac. Sci. Univ. Tokyo, Sect. IA, 28, (1981), 331-413.

[8] T. Kawai, On the theory of Fourier hyperfunctions and its applications to partial differential equations with constant coefficients, J. Fac. Sci. Univ. Tokyo, Sect. IA, 17 (1970), 467-517.

[9] H. Komatsu, Ultradistributions, II, The kernel theorem and ultradistributions with support in a submanifold, J. Fac. Sci. Univ. Tokyo, Sect. IA, 24 (1977), 607-628.

[10] M. Sato, T. Kawai and M. Kashiwara, Hyperfunctions and pseudo-differential equations, Lect. Notes in Math. 287, Springer, (1973) 265-529. 
